\title{
Efectos de dos Protectores Superficiales en las Propiedades de Tableros de Madera después de un Año de Exposición a la Intemperie
}

\author{
Rose Marie Garay \\ Universidad de Chile, Facultad de Ciencias Forestales, Departamento Ingeniería de la Madera, \\ Casilla 9206, Santiago-Chile (e-mail: rgaray@uchile.cl)
}

\begin{abstract}
Resumen
Se evaluaron propiedades físicas, mecánicas y estéticas de tableros estructurales comerciales contrachapados (plywood) y tableros de virutas orientadas (OSB). Estos estaban recubiertos con dos protectores superficiales comerciales para madera que no forman película, y se mantuvieron a la intemperie durante un año en campos de prueba, según norma ASTM D 1006:1973. Se determinó estadísticamente la pérdida de propiedades mecánicas y se evaluó cómo se ven influenciadas las propiedades físicas y estéticas por el tiempo de exposición. Las conclusiones principales fueron que la protección de los recubrimientos utilizados no fue efectiva, no se cumplió la expectativa, de que los no formadores de película protejan la superficie y que no se presenten los defectos típicos de agrietamiento, erosión y decoloración. No se observó diferencias cualitativas entre ambos protectores, por lo que no se justifica el mayor precio de uno de ellos.
\end{abstract}

Palabras clave: propiedades de tableros, tableros de madera, durabilidad, protectores superficiales

\section{Effects of Superficial Protectors on the Properties of Wooden Boards after a Year of Exposure to the Environment}

\begin{abstract}
Physical, mechanical and aesthetic properties were evaluated on structural boards as plywood and oriented strand board (OSB). The boards were covered by two commercial superficial protectors for wood that do not form films, and were maintained outside with no other protection during one year, in test field, according to the ASTM norm D1006:1973. Loss of mechanical properties was statistically determined and the influence of exposure time on the physical and aesthetic properties were evaluated. The main conclusions were that the protection of the used coverings was not effective and the main hypothesis that the non-forming film protect the surface and that typical defects of cracking, erosion and discoloration do not appear, was not proved. It was not observed qualitative differences between both protectors, so the higher price of one of them is not justified.
\end{abstract}

Keywords: board properties, wooden boards, superficial protectors, durability, weathering 


\section{INTRODUCCIÓN}

La protección de la madera y tableros expuestos al exterior es una preocupación mundial que algunos autores (Feist, 1988; Dieguez, 1997; Beall, 2002; William et al., 2001, Deglise, 2005; Garay, 2006; Garay, 2007a; Garay, 2007b; Garay et al., 2008) han estudiado durante años. Se han usado productos eficaces en su duración frente a agentes de degradación, sin embargo éstos son altamente tóxicos y poco amigables con el ambiente. Actualmente la búsqueda se centra en productos menos tóxicos e igualmente eficientes. Los tableros OSB son paneles formados a base de partículas llamadas "strands" (hebras u hojuelas), las cuales se orientan en forma mecánica o electrostática, formando capas delgadas del tablero. De esta manera, el producto puede estar formado por tres o cinco capas dispuestas perpendicularmente entre sí. Las hojuelas son encoladas y prensadas en caliente, empleándose una resina fenólica, dadas las características de uso estructural y exteriores de estos tableros (APAWOOD, 2005). El tablero contrachapado se obtiene mediante encolado de chapas de madera superpuestas de modo que sus fibras formen un ángulo determinado, generalmente recto. Normalmente están compuestos por chapas impares, con el fin de equilibrar su estructura y comportamiento, y facilitar su curvado. queda caracterizado por la especie o grupo de especies de madera empleadas para sus chapas, la calidad de las chapas y el tipo de encolado (APAWOOD, 2005)

La inquietud por encontrar productos más eficientes fue mostrada por ejemplo en un ensayo presentado por Quimunsa (2008), en que se compara la inyección de un protector orgánico y uno base agua, se concluye que la elección de uno u otro no depende de factores técnicos, dado que ambos productos responden adecuadamente, sino más bien a consideraciones toxicológicas y de impacto ambiental.

Durante 15 años, la autora de este trabajo, ha evaluado diversos tipos de protectores, demostrando en varios casos que existen problemas estéticos por resolver Garay (2006 y 2007a). A partir de Garay (2007b), se observó que los protectores no formadores de películas tipo lasur pigmentados, mostraban menor daño en los tableros, luego de dos años de exposición. Se planteó este nuevo experimento para probar si los no formadores de película aplicados en caras y cantos disminuyen los daños en tableros intemperizados. En Garay et al. (2008), se estudió el efecto de condiciones severas de exposición a humedad relativa y temperatura sobre tableros con recubrimientos, entre ellos un protector superficial que no forma película (lasur), los resultados mostraron un buen comportamiento del lasur pigmentado utilizado, sin embargo, quedó la inquietud de conocer el comportamiento de un lasur sin pigmento, pero con filtro solar, con la intención de ser propuesto como una solución de protección inicial que actúe como base imprimante en el tablero, esa fue la motivación para continuar investigando sobre estos productos. Estos son los resultados que se presentan en este estudio.

La radiación solar ultravioleta, es la principal causante de la degradación de las superficies de madera y de los recubrimientos. La luz ultravioleta transmitida inicia reacciones fotoquímicas en la superficie de la madera resultando un cambio de color y falla en la adhesión entre madera y recubrimiento (Garmendia, 2003). El inicio de las reacciones de fotooxidación se atribuye a la absorción de la radiación UV por grupos cromóforos. Los recubrimientos transparentes normalmente utilizados son sensibles a la fotooxidación por la luz ultravioleta. Según Garmendia (2003), la protección contra los efectos de la luz ultravioleta puede ser alcanzada con el uso de absorbentes UV, antioxidantes y HALS (Hindered Amine Light Stabilizers) Compuestos que inhiben la degradación del recubrimiento que ya posee radicales. Por su parte los pigmentos, actúan como estabilizantes ultravioleta, pero a niveles más altos forman sistemas opacos.

Según la descripción de Dieguez (1997), estos lasures están constituidos por resinas alquídicas modificadas al aceite o uretanadas. Ambas tienen los inconvenientes de absorber radiaciones U.V, que degradan el producto. Los disolventes empleados son hidrocarburos alifáticos y aromáticos. Se emplean disolventes orgánicos, para evitar goteos y otros inconvenientes. Es posible encontrar como ligantes soluciones de resinas alquídicas, largas en aceite o ácidos grasos secantes. Estas resinas pueden estas modificadas (Rodríguez, 2004). Los pigmentos más adecuados son óxidos de hierro transparentes, por ser los más sólidos a la luz. Los principios activos mas empleados son, entre los insecticidas, los piretroides (permetrinas y cipermetrinas); y entre los fungicidas, las isothiazonas y 
derivados de los enzimidazoles. En las fichas técnicas de los protectores, pero con pigmento, se promueve su acción protectora frente a decoloración por radiación UV, su acción hidrorepelente, reduciendo el paso de vapor de agua entre el interior y exterior de la madera, su acción fungicida contra hongos y su acción insecticida. Al trabajar a poro abierto y no formar película sobre la madera, permite que ésta respire. Se señala que las propiedades de los productos se mantienen hasta por 6 años. Se eligió dos productos comerciales que ofrecen similares prestaciones, diferenciándose en el precio de venta, menor (P1) y mayor valor (P2). Se intentará probar sí las prestaciones ofrecidas son efectivas.

El objetivo de este estudio es evaluar el comportamiento de dos lasures comerciales, aplicados sobre tableros contrachapados y OSB sometidos a envejecimiento natural. Se mide la perdida de color, brillo, erosión y grietas, absorción de agua e hinchamiento en espesor, además de las propiedades mecánicas de flexión y tracción perpendicular.

\section{METODOLOGÍA}

Se utilizaron un total de 8 tableros estructurales. 4 tableros contrachapado, de dimensiones $1,22 \mathrm{~m} . \mathrm{x}$ $2.44 \mathrm{~m}$. $\times 0,015 \mathrm{~m}$, de fabricado por Arauco S.A. en 5 chapas con madera de Pino Radiata y fenol formaldehído. 4 tableros OSB de dimensiones $1,22 \mathrm{~m} \times 2,44 \mathrm{~m} . \times 0,015 \mathrm{~m}$, fabricado por Louisiana Pacific con maderas de bosque nativo en mezcla con pino radiata y adhesivos fenol formaldehído y Metil di isocianato (MDI). Protectores para madera no formadores de película (P1 y P2). Los protectores de madera utilizados fueron aplicados con brocha a dos manos, su composición química contiene como aditivos Biocidas en base a DIURON (3-(3,4-dichlorophenyl)-1,1-dimethylurea) y carbemacina. Insecticida Permetrina (Piretroide) y/o Ciflutrina (Piretroide). La $\beta$-ciflutrina es un compuesto de baja toxicidad que pertenece al grupo IV de insecticidas, ligeramente tóxico, tiene acción estomacal y de contacto, no tiene actividad sistémica, y controla áfidos, coleópteros, lepidópteros, entre otros insectos (BAYER, 2008). El aditivo incorporado como filtro UV es un óxido de hierro transparente.

Se dimensionaron plantillas de $0,25 \times 2,44 \mathrm{~m}$, se aplicaron los protectores según especificaciones técnicas de fabricantes en caras y cantos de los tableros y se acondicionaron. Se expusieron las plantillas para tiempo 1 (T1), fueron instalados en exposición Norte (N) y Sur (S) según norma ASTM D1006 (1973), en la comuna de La Pintana, Santiago, Chile en el mes de septiembre y retirados 1 año después. Se determinaron propiedades de tableros OSB y contrachapado a tiempo cero (T0) y después de intemperie durante 1 año. Las propiedades analizadas cumplieron con los requisitos de selección y número de probetas establecidos en cada norma de ensayo. Estas fueron: Densidad (UNE-EN 323, 1993). Contenido de Humedad (UNE-EN 322, 1993). Absorción de humedad e hinchamiento en espesor (UNE-EN 317, 1993). Módulo de ruptura y elasticidad en flexión (UNE-EN 310: 1993). Resistencia a la tracción perpendicular a las caras del tablero (UNE-EN 319, 1993). Resistencia a esfuerzos de cizalle (UNE-EN 314-1 y 2, 1993). Las propiedades estéticas se basaron en evaluaciones visuales de decoloración, brillo, erosión y grietas, según metodología habitual en recubrimientos. Los tableros fueron acondicionados dos semanas en condiciones de laboratorio (Fernández-Golfín y Diez Barra, 1996), luego fueron extraídas las probetas para cada ensayo.

\section{RESULTADOS}

Con el análisis estadístico de los datos se estableció la incidencia de los factores: tipo de exposición (norte o sur) y tipo de recubrimiento (P1 y P2) y la interacción entre ambos. Se realizó un análisis bifactorial para las propiedades físicas y mecánicas de los tableros al tiempo 1 (un año de exposición) con diseño experimental en arreglo bifactorial, conforme al modelo de Montgomery, y posteriormente un análisis de varianza (ANDEVA) y para la comparación entre el tiempo cero (sin intemperización) se utilizó un diseño completamente al azar (DCA), modelo de efectos fijos. El análisis estadístico en ambos casos fue con un nivel de confianza del 99\% (Tabla 1). En la Tabla 1, Factor A: Efecto de la exposición (norte o sur); Factor B: Efecto del recubrimiento (P1 y P2) Interacción: Efecto de interacción entre exposición y recubrimiento. Tiempo Cero: Diferencia significativa del factor estudiado entre el tiempo (T1) y el tiempo cero (T0). *Para esta propiedad, debido al deterioro del tablero, el ensayo no pudo realizarse. 
Tabla 1: Resumen de la información entregada por el análisis estadístico de los datos

\begin{tabular}{|c|c|c|c|c|}
\hline Propiedad & Factor A & Factor B & Interacción & Tiempo cero \\
\hline OSB Densidad & $\mathrm{NO}$ & $\mathrm{NO}$ & $\mathrm{NO}$ & $\mathrm{NO}$ \\
\hline Contrachapado Densidad & $\mathrm{NO}$ & $\mathrm{NO}$ & $\mathrm{NO}$ & $\mathrm{NO}$ \\
\hline OSB Hinchamiento a las 2 hrs. & $\mathrm{SI}$ & $\mathrm{NO}$ & $\mathrm{NO}$ & $\mathrm{SI}$ \\
\hline Contrachapado Hinchamiento a las 2 hrs. & $\mathrm{NO}$ & $\mathrm{NO}$ & $\mathrm{NO}$ & $\mathrm{NO}$ \\
\hline OSB Hinchamiento a las 24 hrs. & $\mathrm{NO}$ & $\mathrm{NO}$ & $\mathrm{NO}$ & $\mathrm{SI}$ \\
\hline Contrachapado Hinchamiento a las 24 hrs. & $\mathrm{SI}$ & $\mathrm{NO}$ & $\mathrm{NO}$ & $\mathrm{SI}$ \\
\hline OSB Absorción a las 2 hrs. & $\mathrm{NO}$ & $\mathrm{NO}$ & $\mathrm{NO}$ & $\mathrm{SI}$ \\
\hline Contrachapado Absorción a las 2 hrs. & $\mathrm{NO}$ & $\mathrm{NO}$ & $\mathrm{NO}$ & $\mathrm{SI}$ \\
\hline OSB Absorción 24 hrs. & $\mathrm{NO}$ & $\mathrm{NO}$ & $\mathrm{NO}$ & $\mathrm{NO}$ \\
\hline Contrachapado Absorción 24 hrs. & $\mathrm{NO}$ & $\mathrm{NO}$ & $\mathrm{NO}$ & $\mathrm{NO}$ \\
\hline Contrachapado Cizalle por tracción & $\mathrm{NO}$ & $\mathrm{NO}$ & $\mathrm{NO}$ & $\mathrm{NO}$ \\
\hline OSB Tracción normal & - & - & - & $\mathrm{SI}$ \\
\hline Contrachapado Flexión estática (MOR) & $\mathrm{NO}$ & $\mathrm{NO}$ & $\mathrm{NO}$ & - \\
\hline Contrachapado Flexión estática (MOE) & $\mathrm{NO}$ & $\mathrm{NO}$ & $\mathrm{SI}$ & - \\
\hline OSB Flexión estática (MOE) & $\mathrm{SI}$ & $\mathrm{SI}$ & $\mathrm{SI}$ & $\mathrm{SI}$ \\
\hline OSB Flexión estática (MOR) & $\mathrm{NO}$ & $\mathrm{SI}$ & $\mathrm{NO}$ & $\mathrm{SI}$ \\
\hline
\end{tabular}

\section{Propiedades Físicas}

Las propiedades físicas aumentan con el tiempo de exposición, Los protectores utilizados no disminuyen la acción de la humedad, el libre paso de ésta se transforma en aumentos de densidad, contenido de humedad e hinchamiento en espesor y el cambio más significativo se observa en absorción de humedad. La Fig. 1 muestra el contenido de humedad $(\mathrm{CH})$. Los valores más altos se alcanzaron al año de exposición en contrachapado con P2, la diferencia entre norte y sur es mínima. Hay aumento con respecto a T0, pero el $\mathrm{CH}$ no supera el $13 \%$, siendo mayor en contrachapado que en OSB. La Fig. 2 muestra las densidades en contrachapados y OSB. El análisis estadístico reveló que no existe significancia de la exposición y del protector. En la Fig. 3, transcurrido un año de exposición los tableros captan mayor cantidad de agua. P1 mostró una menor absorción que P2. El tablero contrachapado presentó una absorción mayor que OSB. El análisis de varianza señaló que la absorción de humedad a las 2 horas, tuvo influencia significativa en contrachapado y en OSB sólo a las 24 horas. En la Fig. 4 se observa que contrachapado presentó menor hinchamiento que OSB. Para esta propiedad, inicialmente los valores de $\mathrm{P} 1$ fueron mayores que $\mathrm{P} 2$, al cabo de 1 año de exposición esta situación se revirtió y fue mayor en exposición sur que en norte para P1. En T1 el hinchamiento a 2 horas en contrachapado no presenta gran diferencia con respecto a 24 horas, no así en OSB. El análisis de varianza indicó que el hinchamiento en espesor tuvo influencia significativa en OSB a las 2 y 24 horas y en contrachapado solo tuvo influencia a las 24 horas.

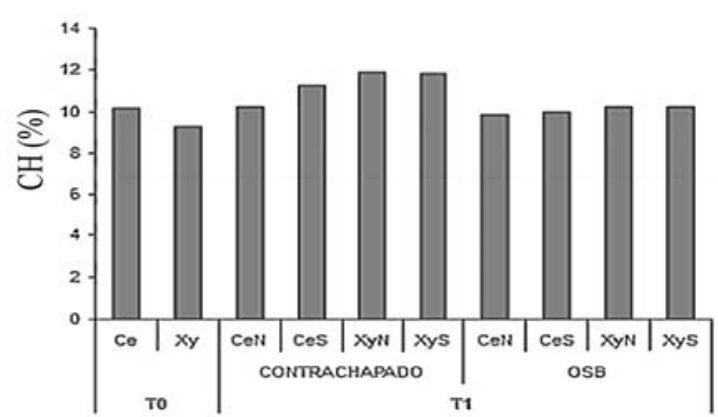

Fig. 1: Contenido de humedad (\%)

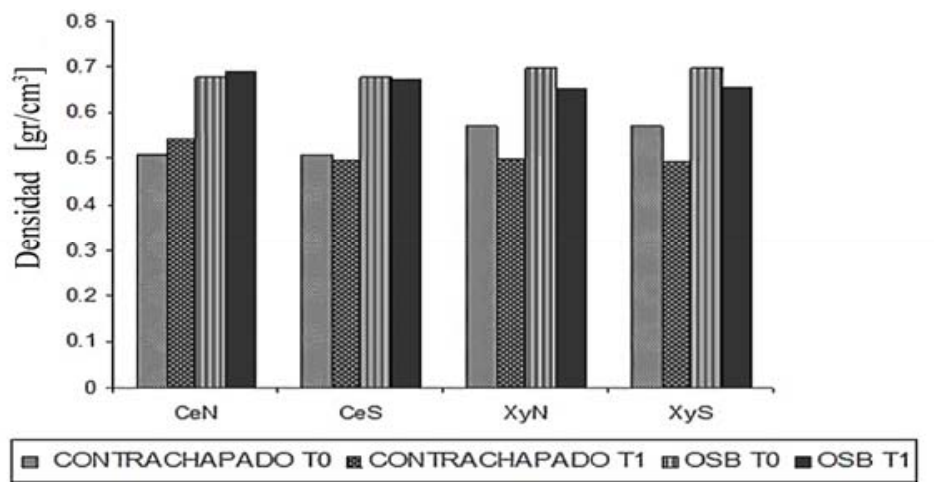

Fig. 2: Densidad $\mathrm{gr} / \mathrm{cm}^{3}$

\section{Propiedades mecánicas}

La resistencia a la flexión, expresada en módulo de ruptura (MOR) muestra disminución para ambos tableros, sin influir en ésta el protector usado. En módulo de elasticidad (MOE) se evidencia un cambio muy notorio entre T0 y T1, un aumento en la plasticidad de los tableros producto del aumento de absorción de humedad. El cizalle por tracción en contrachapado muestra la alta resistencia de la unión encolada en contrachapados, los bajos valores observados en tracción perpendicular de OSB muestra la baja resistencia interna de la unión encolada en OSB. 


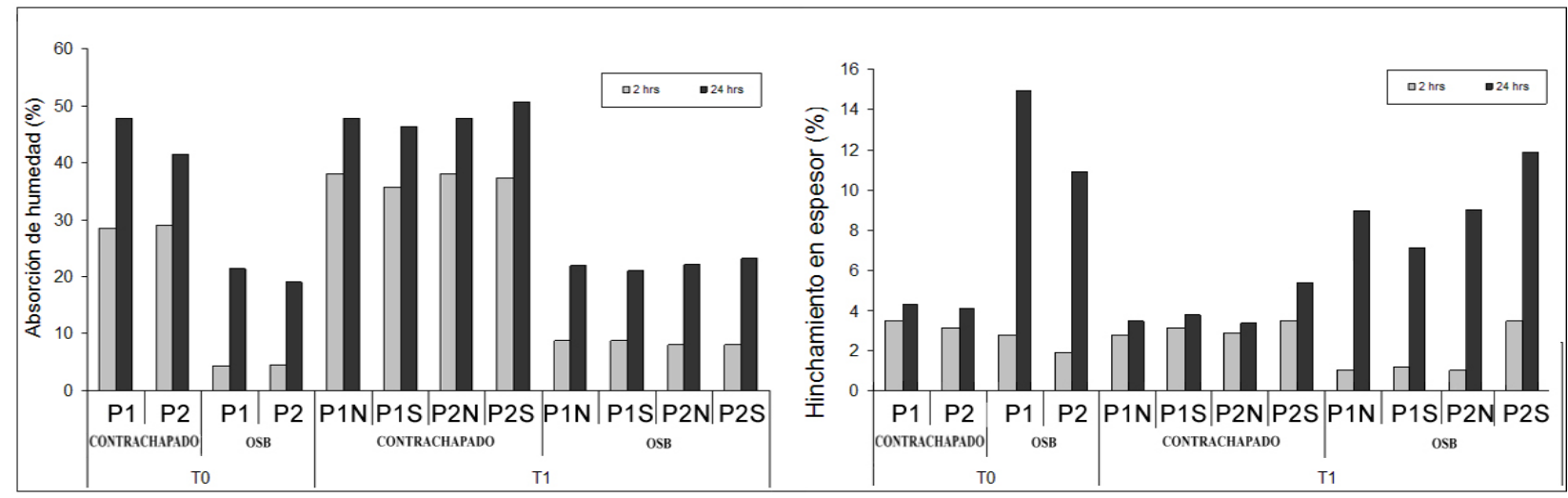

Fig. 3: Absorción de humedad (\%)

Fig. 4: Hinchamiento en espesor (\%)

En la Fig. 5 se compara MOR y en la Fig. 6 se compara MOE. Contrachapado es más resistente que OSB, antes y luego de 1 año de exposición. En el análisis estadístico se observó que para contrachapado no hubo influencia significativa de ningún factor y en OSB hubo influencia del tipo de protector. Se observó diferencias menos notorias utilizando P2 tanto en exposición norte como en sur. P1S presentó el mayor valor para contrachapado y P2S para OSB. En la Fig. 7 se observó que en cizalle por tracción, los valores sobrepasan $15 \mathrm{Kg} / \mathrm{cm}^{2}$, a excepción de P2N. La Fig. 8 muestra los resultados en tracción normal para OSB. Los valores de $\mathrm{P} 2 \mathrm{~N}$ no se grafican, ya que, todas las probetas fallaron antes del ensayo indicando falla de la unión adhesiva interna generada por la humedad presente.

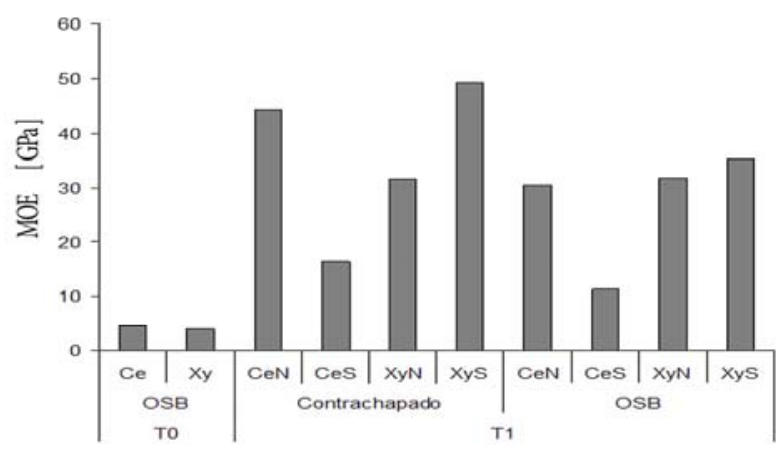

Fig. 5: Módulo de ruptura $\left(\mathrm{Kg} / \mathrm{cm}^{2}\right)$

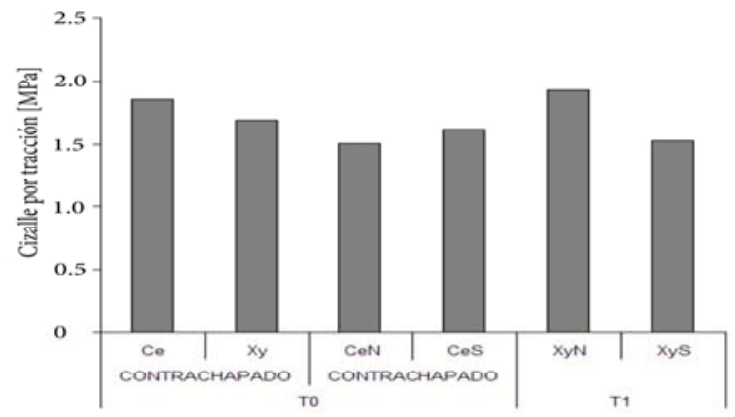

Fig. 7: Cizalle por tracción contrachapado

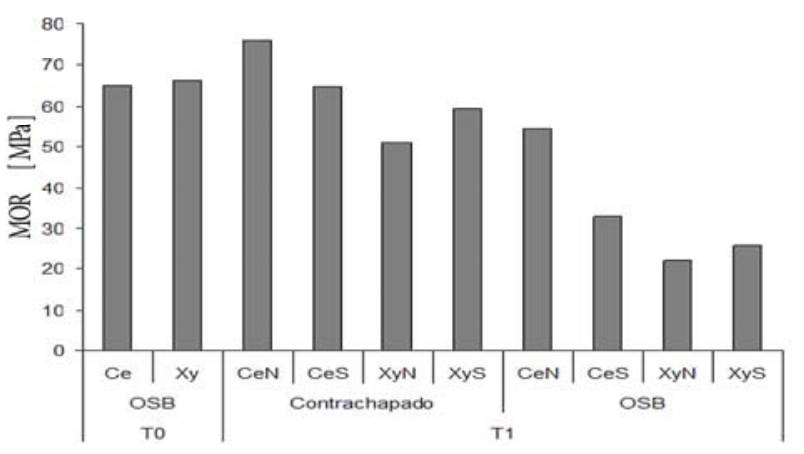

Fig. 6: Módulo de elasticidad $\left(\mathrm{Kg} / \mathrm{cm}^{2}\right)$

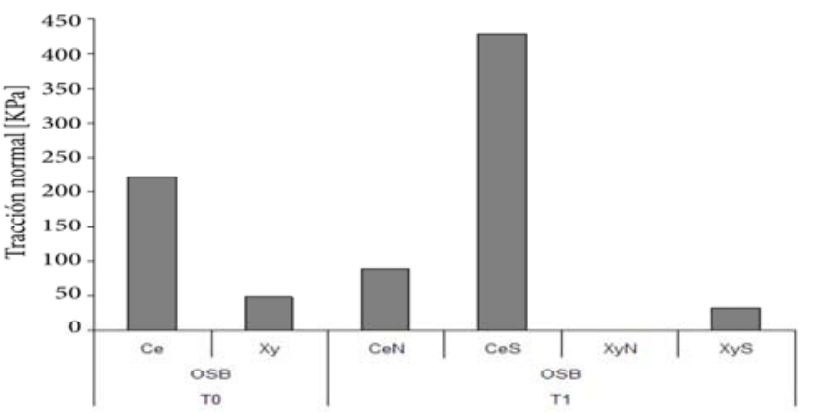

Fig. 8: Tracción normal en OSB

\section{Propiedades estéticas}

Al completarse el año de exposición (T1) ya no existía presencia de los protectores aplicados, éstos habían sido degradados por las condiciones climáticas, el sol, la lluvia y el viento fueron lixiviando el protector hasta su completa desaparición. P1N protegió menos frente a grietas y hongos de mancha que P2N. En exposición sur los hongos de mancha fueron muy_agresivos y los protectores no | funcionaron. Se observa fuerte erosión en contrachapado Sur y OSB Sur. Alabeos y agrietamiento 
superficial fueron observados en contrachapados Norte. En OSB Sur se apreció deformación en cantos y en OSB Norte erosión y desprendimiento de hojuelas (Fig. 9). Cabe hacer notar que en un trabajo anterior de Garay (2005) se evaluaron tableros sin protección bajo condiciones severas de exposición y los resultados estéticos observados en ambos estudios son muy similares, comprobándose que la acción protectora de estos lasures transparentes no funciona.

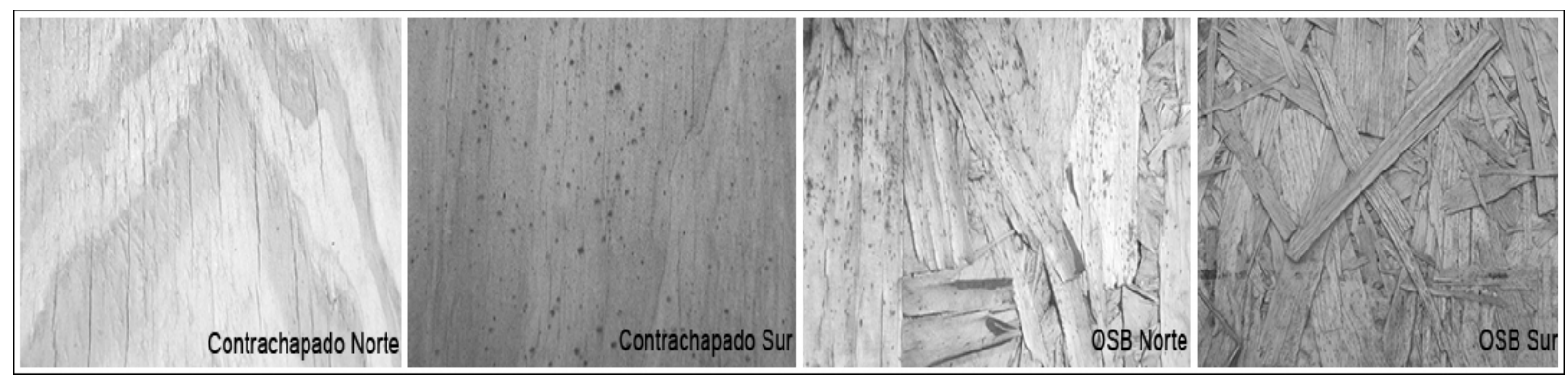

Fig. 9: Aspecto visual de los tableros después de 1 año de exposición

\section{DISCUSIÓN}

Actualmente la composición química de protectores superficiales consideran los Absorbentes UV con estabilizadores a la luz de Aminas estericamente impedidas (HALS), compuestos que han sido investigados por Schaler y Roget (2008), Deglise (2005) y Garmendia (2003). Sin embargo, éstos no responden adecuadamente en materiales porosos como la madera, en este sentido, una estrategia de protección investigada, pero que posee detractores, es utilizar estabilizadores de lignina aplicados como pretratamiento. Los protectores investigados en este trabajo poseen óxido de hierro, que actúan como absorbentes de UV ello dificulta a la luz alcanzar el sustrato. Sin embargo, el medio más eficaz de proteger la madera del daño de la luz UV es la opacidad de la capa de protección, la cual depende de los pigmentos utilizados. La relevancia de este estudio radica en que los tableros estructurales son diseñados para aplicaciones ingenieriles como componentes estructurales donde las propiedades de diseño, tracción, compresión, corte, flexión y comportamiento en uniones tiene importancia significativa. Además de las especies de madera a emplear, el grado o calidad de cada una y el set de exigencias de la unión encolada, los paneles estructurales poseen una serie de exigencias definidas en el Estándar PS1 -07 en donde se encuentran todos los tipos y grados aplicables (APAWOOD, 2008).

Los resultados permiten entender que los protectores superficiales utilizados, así como el tipo de exposición no son estadísticamente significativos $(99 \%)$ al evaluar su efecto sobre las propiedades físicas y mecánicas de los tableros, éstos se mantienen dentro de rangos de calidad aceptables de sus propiedades. Al igual que en el estudio de Rao (1999), se demuestra que las propiedades hidrotérmicas afectan fuertemente el transporte y la acumulación de humedad en la cubierta de techumbres, base para pisos y forros exteriores, que implicarán cambios en las propiedades de los tableros. La heterogeneidad del espesor del panel puede deformar la cubierta de una techumbre. La madera protegida por un recubrimiento y expuesta al aire libre está propensa a cambios de humedad, que inducen tensión dentro de los tableros. Para cumplir con su papel protector, un acabado tiene que seguir los cambios dimensionales del substrato sin agrietarse y descascararse. La degradación se relaciona más a la fractura de la zona interfacial madera /recubrimiento que a una disminución de la cohesión de la película (Deglise, 2005). Un interesante análisis de la situación de empleo de OSB y contrachapado en EE.UU. ha sido publicado por Fisette (2005) en este documento se plantea los cuestionamientos de uso que se generan por la incorporación de los tableros OSB en la construcción en Norteamérica, quedando de manifiesto los problemas de humedad asociados a estos tableros. La duración del acabado de un recubrimiento orgánico para la madera al exterior depende fundamentalmente de tres grandes factores, la inestabilidad superficial de la madera, la intemperización causada por agentes atmosféricos y el mecanismo de envejecimiento, que se produce en los polímeros sólidos por degradación biótica y abiótico (Dieguez, 1997).

La calidad de las lasures está ligada a la cantidad de resina de la capa de impregnación que penetra en la madera y del espesor de la película seca. Del mismo modo, la transición vítrea, es hoy en día, de la máxima utilidad en el estudio del envejecimiento de los recubrimientos. Los valores de la 
transición vítrea marcan la elasticidad de las resinas. Las variaciones en el comportamiento reológico de los polímetros se estudian considerando la temperatura. Para ello se utiliza el parámetro de modulo de relajación que es función de las deformaciones del cuerpo con la temperatura y cuyo valor se obtiene empíricamente (Dieguez, 1997), este concepto permite comprender el juego e interfase que ocurre entre recubrimiento y sustrato que deben permanecer unidos entre sí.

Fernández-Golfín y Diez Barra (1996), investigaron el comportamiento reológico de tableros de partículas con cubierta melamínica fabricados con urea formaldehído, concluyendo que la aplicación de revestimientos de este tipo reducía la fluencia relativa y aumentaba el tiempo hasta la rotura. Se señala que en los aglomerados las tensiones dentro del material dependen de factores locales como la orientación de las partículas, la densidad, el tipo y contenido de adhesivos. Además se incrementan por factores externos como las condiciones climáticas circundantes. Si además de la carga intervienen altas temperaturas, se produce una plastificación de la lignina que hace que la fluencia aumente. En este estudio, afirma que el tablero MDF presenta una mayor propensión hacia la fluencia conforme la humedad relativa aumenta. Se comprobó también una mayor fluencia en tableros MDF sometidos a condiciones cíclicas semanales de $20^{\circ} \mathrm{C} / 30 \%-20^{\circ} \mathrm{C} / 85 \%$. En otro trabajo de Fernández y Diez (1998), se estudió el proceso de desorción en MDF el que se efectúa más rápidamente que el de adsorción, si bien cuantitativamente es menor, por lo que la repetición de ciclos de adsorción/desorción lleva a un incremento de la Humedad de Equilibrio Higroscópica (HEH) media del tablero. Aunque los tableros MDF no son estructurales, la investigación realizada por Fernández y Diez (1998), permite comprender los fenómenos asociados a su comportamiento frente a cambios de humedad y temperatura y en este sentido existen similitudes a las observadas en los tableros OSB y contrachapados. Principalmente en relación a aumento de absorción de humedad después de 1 año de exposición, simultáneamente disminución de hinchamiento en espesor, efecto que muestra a los tableros estructurales capaces al mismo tiempo de absorber agua y ser estables dimensionalmente, ya que el aumento en espesor es proporcionalmente menor se deduce también que la desorción es más rápida que la adsorción.

\section{CONCLUSIONES}

Aunque hay pérdida de las propiedades mecánicas de los tableros, se mantienen dentro de parámetros aceptables por normas de ensayo. Las propiedades físicas de hinchamiento y absorción se ven muy influenciadas por la intemperización, siendo inaceptable por normas de ensayo la absorción de humedad obtenida. Los protectores no ofrecieron niveles de protección adecuados y los daños estéticos son severos. En general, los factores Efecto de la exposición (norte o sur); Efecto del recubrimiento (P1 y $\mathrm{P} 2$ ) y Efecto de interacción entre exposición y recubrimiento no resultaron estadísticamente significativos para explicar las diferencias de propiedades observadas. Sí se constató diferencia significativa del factor tiempo estudiado T1 y T0.

\section{REFERENCIAS}

APAWOOD. Performance Standard for Wood-Based Structural-Use Panels. Covers Voluntary Product Standard PS 2-04 and PS 1-07 describes the requirements for producing and testing woodbased structural-use panels. Updated May 2008. en línea [Consulted 28th may 2008]. http://www.apawood.org/level c.cfm?content=pub ply libmain.

ASTM D 1006; Test Methods for Conducting Exterior Exposure Tests of Paints on Word (1973).

Bayer; http://www.environmental.bayercropscience.cl/db_images/folletos/archivos/Ficha.pdf. (2008)

Beall, F.; Preservación por Degradación Térmica. Forest Products Laboratory, University of California, Richmond, CA. Primer Congreso Chileno en Ciencias Forestales. Universidad de Chile (2002).

Deglise, X.: La protección superficial de la madera: tendencias actuales y perspectivas futuras. Actas en CD room, IV Congreso de Protección de la Madera, San Sebastián, España. 14 a 17 de junio (2005).

Dieguez, M.J.; Acabado de la madera. Duración al exterior y temperatura de transición vítrea. AITIM: 186(Marzo /Abril), 60-64 (1997). 
Feist W.; Role of pigment concentration in the weathering of semitransparent stains. Forest Prod. J.: 38(2), 41-44 (1988).

Fernández-Golfín Seco, J.I. y M. Diez Barra; Comportamiento reológico de los tableros MDF sometidos a condiciones de humedad relativa: comparación con el tablero de partículas. AITIM No 180(Abril/Mayo), 75-80 (1996).

Fernández, J. y M. Diez; Efecto de las condiciones ambientales sobre las propiedades físicomecánicas de tableros MDF. Revista Investigación Agraria: 1 y 2, 29-41 (1998).

Fisette, P.; Choosing Between Oriented Strandboard and Plywood. Building Materials and Wood Technology. University of Massachusetts, Amherst (2005). http://www.umass.edu/bmatwt/ publications/articles/osb vs plywood.html

Garay, R.; Evaluación del comportamiento físico y mecánico de dos tipos de tableros estructurales bajo condiciones extremas de temperatura y humedad relativa. Actas in extenso en CD-room. Congreso mundial de IAWS. International Acaderny y $X$ Reunión de Investigación y Desarrollo de Productos Forestales. Concepción, Chile (2005).

Garay, R.; Recubrimientos superficiales para uso exterior. Revista Protecma No 29. España. Febrero. 16- 22 (2006).

Garay, R.; Tableros Contrachapados. Facultad de Ciencias Forestales. Universidad de Chile. Santiago, Chile. 172 Pág. (2007a)

Garay, R.: Impregnantes tipo lasur para la protección de la madera y tableros. Abril 2007. AgroCiencia: 23(1), 25-36 (2007b).

Garay, R.; H. Poblete y T. Karsulovic; Evaluation of oriented strandboards and plywood subjected to severe conditions of relative humidity and temperature. FP J (Accepted for publication (2008).

Garmendia, I. Mecanismos de Degradación y Sistemas de Protección en Barnices y Pinturas Frente a Agentes Externos. Revista PROTECMA N5. [en línea] Disponible en www.esinal.es. (2003).

Quimunsa. Comparación entre la inyección de un protector orgánico y un protector base agua. Revista Protecma No 38. España Mayo. 28-29 (2008).

RAO, J.; Conceptual refence database for building envelope research (1999). http://alcor.concordia .ca/raojw/crd/essay/essay00129.html

Rodríguez, B. Curso sobre Lasures. Ligantes (vehículos) para lasures.18p. En Jornada técnica sobre lasures para madera, Valencia (España), 10 marzo (2004).

Schaler, C. y D. Roget; Protección de la Madera frente al sol. Empleo optimizado de absorbentes y desgaste del sustrato. Revista Protecma № 39: España Agosto. 16-21 (2008).

UNE-EN 310; Determinación del Módulo de Elasticidad en Flexión y la Resistencia a la Flexión (1993).

UNE-EN 314; Calidad de encolado tableros contrachapados. Parte I y Parte II (1993).

UNE-EN 317; Determinación de Hinchamiento en espesor y absorción de humedad (1993).

UNE-EN 319; Determinación de tracción perpendicular a las caras del tablero (1993).

UNE EN 322; Determinación del Contenido de Humedad (1993).

UNE-EN 323; Tableros derivados de la Madera. Determinación de la Densidad (1993).

Williams, R.S.; M.T. Knaebe, P.G. Sotos y W.C. Feist; Erosion rates of wood during natural weathering. Part I Effects of grain angle and surface texture. Forest Products Laboratory. USDA.Wood Fiber Sci.: 33(1), 31-42 (2001). 